\title{
Direct insight into diamonds formation from deep C-O-H fluids.
}

\section{MARIA LUCE FREZZOTTI}

\author{
Università di Milano Bicocca DISAT CSS1
}

Presenting Author: maria.frezzotti@unimib.it

In subduction zones, diamond formation is mainly regulated by oxidized $\mathrm{C}-\mathrm{O}-\mathrm{H}$ fluids delivered by decarbonation and dissolution reactions at high $\mathrm{P}$ and $\mathrm{T}$ (i.e., $600-900^{\circ} \mathrm{C}$; $3-5 \mathrm{GPa}$ ). To provide direct insight into carbon speciation in deep metamorphic fluids, I present Raman microspectroscopy data on the structure and composition of carbon-bearing phases in fluid inclusions trapped in metamorphic rocks from the diamondbearing ophiolitic slice of Lago di Cignana (western Alps, Italy) $[1,2]$. Analysis of fluid inclusions has the advantage of examining a direct sample of the medium in which diamond formed. Data show the presence of carboxylic acids and carbonate and bicarbonate ions in deep fluids. Results suggest that the decomposition of carboxylic acids can induce diamond nucleation on the reduction of carboxyl groups. Structural intermediates could allow diamond growth similarly to models of diamond nucleation by metastable molecular precursors [3]. The present study provides direct evidence that dissolved inorganic carbon can spontaneously evolve to organic species without biologically catalyzed processes. Results suggest a different nucleation mechanism for diamonds in which the nucleation energetic barrier could be lower than that generally assumed by classical models.

[1] Frezzotti, Selverstone, Sharp, Compagnoni (2011) Nature Geoscience 4, 703-706.

[2] Frezzotti (2019) Nature Communications 10, 4952.

[3] Gebbie et al. (2018) PNAS 155, 33, 8284-8289 